\title{
Long-Term Outcomes of Pediatric Meningioma Surgery: Single Center Experience with 23 Patients
}

\author{
Ilkay ISIKAY ${ }^{1}$, Sahin HANALIOGLU1 ${ }^{1}$, Firat NARIN² ${ }^{2}$ Ibrahim BASAR ${ }^{3}$, Burcak BILGINER ${ }^{1}$ \\ ${ }^{1}$ Hacettepe University School of Medicine, Department of Neurosurgery, Ankara, Turkey \\ ${ }^{2}$ Van Yuzuncuyil Training and Research Hospital, Neurosurgery Clinic, Van, Turkey \\ ${ }^{3}$ Dicle University School of Medicine, Department of Neurosurgery, Diyarbakir, Turkey \\ This study has been presented at the $43^{\text {rd }}$ Annual Meeting of the ISPN between 4 and 8 October 2015 at Izmir, Turkey \\ Corresponding author: Ilkay ISIKAY isikay@hacettepe.edu.tr
}

\section{ABSTRACT}

AIM: To evaluate the long-term outcomes and prognostic variables in a surgical cohort of pediatric meningiomas treated in a single institution.

MATERIAL and METHODS: Medical records and follow-up notes of 23 pediatric patients aged $<18$ years (12 male and 11 female; mean age on presentation, $13.1 \pm 4.4$ years) harboring 27 meningiomas operated between 1994 and 01/2019 at Hacettepe University Ihsan Dogramaci Children's Hospital were evaluated.

RESULTS: One patient had neurofibromatosis (NF) type 1, and five patients had NF2. Tumors were most commonly located in the convexity $(n=6)$ and parasagittal or falcine $(n=6)$. Gross total resection was performed in $70.4 \%$ of cases. WHO grade I tumors accounted for $56 \%$ of all cases, whereas high-grade meningiomas accounted for $44 \%$ (33\% grade II, $11 \%$ grade III). The mean follow-up duration was $10.3 \pm 7.7$ years. Three patients $(13 \%)$ died during follow-up, and $76.2 \%$ of the patients had favorable outcome (Glasgow Outcome Scale > 3) during the last follow-up assessment. Ten patients $(43.5 \%)$ had relapse. In univariate analysis, low histological grade $(p=0.030)$ and gross total resection $(p=0.024)$ were associated with favorable outcome. The 10-year overall survival rate was $86 \%$.

CONCLUSION: Meningiomas in the pediatric age group are surgically treatable tumors with fairly good outcomes. However, relapses are common even for low-grade tumors; therefore, long-term surveillance and aggressive treatment are needed.

KEYWORDS: Meningioma, Childhood, Surgery, Outcome, Recurrence

ABBREVIATIONS: CNS: Central nervous system, DWI: Diffusion-weighted imaging, GOS: Glasgow Outcome Scale, ICP: Intracranial pressure, NF: Neurofibromatosis, WHO: World Health Organization

\section{INTRODUCTION}

$\mathrm{M}$ eningiomas are a relatively rare tumor type in pediatric and adolescent patients. In adults, meningiomas are the most common primary intracranial tumors that constitute approximately $30 \%$ of the cases, whereas it only makes up $0.4 \%-4.6 \%$ cases in the pediatric or adolescent age group (15). Because of its rarity, knowledge about this tumor type is derived mostly from small case series and individual reports. A systematic review of published series identified epidemiologic features, clinical characteristics, survival outcomes, and main differences of this rare tumor type from its adult counterparts (15).

\begin{tabular}{llll}
\hline Ilkay ISIKAY & (1) : 0000-0001-7790-4735 & Ibrahim BASAR & (1) : 0000-0003-3674-4864 \\
Sahin HANALIOGLU (D) : 0000-0003-4988-4938 & Burcak BILGINER & (1) : 0000-0001-9667-3709 \\
Firat NARIN & (1) : :0000-0002-5985-4460 & &
\end{tabular}


In contrast to other childhood central nervous system (CNS) tumors $(3,13)$, prognosis and survival are better for pediatric or adolescent meningiomas $(10,15,18)$. There is paucity of reports on pediatric meningioma cases that report longterm survival data in the magnetic resonance imaging (MRI) era. Thus, this study aimed to identify epidemiological and clinicoradiological features, short- and long-term outcomes, as well as prognostic factors affecting favorable results in pediatric meningioma based on a 25-year experience of a single center.

\section{MATERIAL and METHODS}

Admission and discharge notes of pediatric patients (patient age less than 18 years at the time of first admission) with intracranial or spinal meningiomas who were surgically treated at Department of Neurosurgery, Hacettepe University Ihsan Dogramaci Children's Hospital between 1994 and 01/2019 were reviewed, and follow-up visit notes were evaluated. The variables analyzed were age, sex, clinical presentation, as well as association with neurofibromatosis, extent of resection, and tumor histology or grade. Simpson grades I-III were accepted as gross total resection, whereas Simpson grade IV was deemed subtotal resection. Radiological workup, particularly the MRI scans of these patients, was reassessed in terms of tumor location, size, and enhancement pattern. For patients whose final follow-up assessments were done in outside centers, the Ministry of Health web-based patient registry and telephone interview were utilized. Final outcome of patients were categorized according to Glasgow Outcome Scale (GOS).

SPSS software (IBM SPSS Statistics for Macintosh, version 23.0; IBM Corp., Armonk, NY, USA) was used for statistical analysis. Descriptive statistics included means and standard deviation for parametric data, median and range for nonparametric, as well as counts and percentages for categorical data. Two group comparisons were performed using Student's t-test for parametric, Mann-Whitney $U$ test for nonparametric, and Chi-square and Fisher's exact tests for categorical variables. Logistic regression was used to assess factors affecting favorable outcome. $\mathrm{p}<0.05$ was considered statistically significant.

\section{RESULTS}

During the specified time period, 27 meningiomas either intracranial or spinal in nature in 23 patients were operated. Four spinal meningiomas in three patients and 23 intracranial meningiomas in 20 patients were detected. The total number of pediatric intracranial tumors operated between 1994 and 01/2019 was 913; thus, the incidence for intracranial meningiomas in pediatric age group was $2.5 \%$. The total number of intracranial meningiomas during the same period was 1533; therefore, study cohort made up $1.7 \%$ of all meningioma cases.

There were 12 male and 11 female patients (Table I). Mean ages \pm SD upon diagnosis for the whole study group, male subgroup, and female subgroup were $13.1 \pm 4.4,13.9 \pm 3.9$, and $12.2 \pm 4.9$ years, respectively. The age at presentation between different sex groups did not differ significantly.
Table I: Characteristics of Study Population

\begin{tabular}{lc}
\hline Category & Value \\
\hline Number of patients (\%) & \\
\hline Male & $12(52.2)$ \\
\hline Female & $11(47.8)$ \\
\hline Mean age at presentation \pm SD (years) & $13.1 \pm 4.4$ \\
\hline Male & $13.9 \pm 3.9$ \\
\hline Female & $12.2 \pm 4.9$ \\
\hline Neurofibromatosis (NF) (\%) & \\
\hline NF type 1 & $1(4.3)$ \\
\hline NF type 2 & $5(21.7)$ \\
\hline
\end{tabular}

Tumor location (\%)

\begin{tabular}{|c|c|}
\hline Superficial & $13(48.2)$ \\
\hline Convexity & 6 \\
\hline Parasagittal & 6 \\
\hline Peri-sylvian & 1 \\
\hline Deep & $3(11.1)$ \\
\hline Pineal & 1 \\
\hline $\begin{array}{l}\text { Mesencephalo-thalamic (ambient } \\
\text { cistern) }\end{array}$ & 1 \\
\hline Lateral ventricle & 1 \\
\hline Skull base & $7(25.9)$ \\
\hline Foramen magnum & 2 \\
\hline Retro-orbital & 3 \\
\hline Anterior cranial fossa & 2 \\
\hline Spinal & $4(14.8)$ \\
\hline \multicolumn{2}{|l|}{ Extent of resection (\%) } \\
\hline Gross total & $19(70.4)$ \\
\hline Subtotal & $8(29.6)$ \\
\hline \multicolumn{2}{|l|}{ Histologic grade (\%) } \\
\hline Grade I & $15(55.6)$ \\
\hline Grade II & $9(33.3)$ \\
\hline Grade III & $3(11.1)$ \\
\hline Mean follow up \pm SD (years) & $10.3 \pm 7.7$ \\
\hline \multicolumn{2}{|l|}{ Outcome (\%) } \\
\hline GOS 1 & $7(33.3)$ \\
\hline GOS 2 & $9(42.9)$ \\
\hline GOS 3 & $2(9.5)$ \\
\hline GOS 4 & - \\
\hline GOS 5 & $3(14.3)$ \\
\hline
\end{tabular}

SD: Standard deviation, GOS: Glasgow outcome scale. 


\section{Presentation}

The presentation patterns of patients were related to tumor locations. The most common presenting symptom was headache $(n=6)$; followed by seizure $(n=5)$; motor weakness in the form of lower extremity monoparesis, paraparesis, or hemiparesis $(n=4)$; proptosis $(n=3)$; imbalance $(n=2)$; extrapyramidal symptoms $(n=1)$, and scalp mass $(n=2)$. Six patients (26\%) had associated neurofibromatosis (NF); one patient had NF1, and five patients had NF2. None of the patients had history of irradiation, but one patient had a concomitant hemophagocytic syndrome and hepatoblastoma.

\section{Tumor Location and Size}

Most of the tumors had superficial locations; six (22\%) were located in cerebral convexity, six (22\%) in parasagittal, and one $(4 \%)$ in perisylvian regions (Figure $1 \mathrm{~A}-\mathrm{F}$ ), which accounted for $13(48 \%)$ of the 27 meningiomas in 23 patients. Skull base tumors were observed in seven (26\%) patients. Tumor invaded the orbita and retro-orbital space extending into the cavernous sinus in three $(11 \%)$ patients. Three of the tumors were located deep in the cerebral hemispheres, namely, the pineal region, adjacent to the midbrain and thalamus (perimesencephalic cistern), and the lateral ventricle. The brainstem was displaced due to juxtaposing tumors (foramen magnum and petroclival meningioma) in two $(7 \%)$ patients. Overall, five (19\%) tumors were infratentorial. Finally, there were four (15\%) tumors that originated from the spinal intradural space in three patients. One patient presented with T1-3 meningioma and four years later de novo T12-L1 meningioma (both papillary type). Remaining two patients presented with T3-6 and T7-8 meningiomas (both psammomatous type) respectively. Out of 23 intracranial tumors, 15 (65\%) were larger than $4 \mathrm{~cm}$ in diameter. The mean tumor size (maximal diameter) was $5.2 \pm 1.9 \mathrm{~cm}$ (median $5 \mathrm{~cm}$; range $2.5-10 \mathrm{~cm}$ ). The mean tumor volume was $59 \pm 71 \mathrm{~cm}^{3}$ (median $31.4 \mathrm{~cm}^{3}$; range $3-293 \mathrm{~cm}^{3}$ ).

\section{Extent of Resection}

Gross total resection was performed in 19 (70.4\%) of the meningiomas at initial surgery. Only a subtotal resection could be achieved in eight $(29.6 \%)$ tumors. All convexity or parasagittal tumors could be excised completely (Simpson grade I-III). The reasons for subtotal resection (Simpson grade IV) were orbital tumors that extended from the bony orbit to retro-orbital space invading the cavernous sinus in three
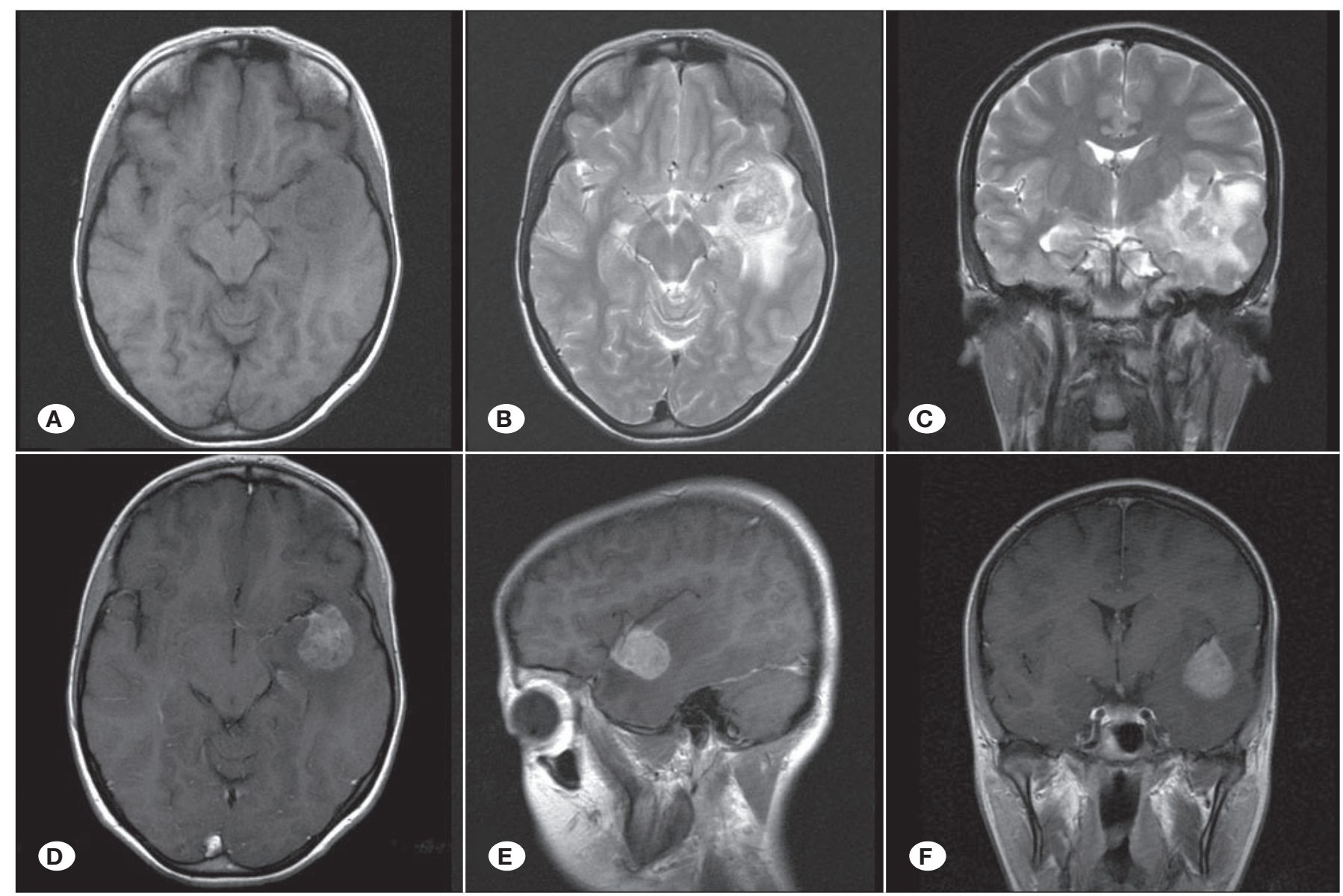

Figure 1: Cranial magnetic resonance image of a 17-year-old girl who presented with seizure shows a left temporal and intrasylvian mass (A). Surrounding tissue exhibits marked edema particularly in the external capsule, posterior limb of internal capsule, and periventricular white matter (B, C). In postcontrast images (D, E, F), enhancement of pia-arachnoid indicates tumoral infiltration. Histopathological examination of the lesion suggested atypical meningioma WHO grade II. 
patients; World Health Organization (WHO) grade II tumors that invaded the brain stem in two patients; WHO grade III tumors that invaded the spinal cord in one patient (two separate tumors located in the thoracic and lumbar dura); and one giant tumor in the frontal basal area.

\section{Surgical Complications}

No operative mortality was observed in this series. Perioperative complications occurred in four patients (17\%). Slight right hemiparesis developed in one patient who had a convexity meningioma over the motor cortex. In a patient with thoracic spinal meningioma, paraparesis worsened postoperatively. Postoperative third nerve palsy developed in a patient who had a tumor originating from tentorial incisura extending to ambient cistern, which displaced the mesencephalon and thalamus. Another patient required ventriculoperitoneal shunting due to hydrocephalus that developed after resection of a skull base meningioma.

\section{Histological Types}

The distribution of WHO grade I, grade II, and grade III meningiomas were $15(56 \%), 9(33 \%)$, and $3(11 \%)$, respectively. Overall, there were 12 (44\%) high-grade tumors. The most common initial histological tumor type was atypical meningioma $(n=8)$, followed by transitional $(n=6)$, meningothelial $(n=4)$, psammomatous $(n=2)$, fibroblastic $(n=2)$, papillary $(n=2)$, rhabdoid $(n=1)$, and clear cell $(n=1)$. One specimen showed both fibroblastic and meningothelial meningioma features. One NF2 patient, who was operated for left frontoparieatal convexity meningioma had transitional histologic type; the same patient was operated after three years for de novo right parietal convexity meningioma which turned up to be rhabdoid type.

\section{Follow-up and Outcome}

Two patients were lost to follow-up immediately after operation. The mean follow-up duration for the remaining
21 patients was $10.3 \pm 7.7$ years (median 9 years; range $0.5-25$ years). During the follow-up period, four patients who underwent gross total resection of the original tumor had recurrences after a mean of 3.8 years. Of the tumors that had been resected subtotally, six had residual tumor progression with a mean duration of 4.0 years (Table II). Relapses $(n=10$; $43.5 \%)$ occurred in $33.3 \%$ of $\mathrm{WHO}$ grade I $(n=5)$ and in $41.7 \%$ of WHO grade II or III $(n=5)$ meningiomas. Mean latencies to relapse for WHO grade I and grade II or III meningiomas were $5.0 \pm 2.9$ years and $2.2 \pm 2.1$ years, respectively $(p=0.067)$. Six patients were reoperated (two of them three times) due to tumor relapse. During the last follow-up, GOS scores were distributed as follows: $\mathrm{GOS}=5$ in seven patients, $\mathrm{GOS}=4$ in nine patients, GOS $=3$ in two patients, and $\mathrm{GOS}=1$ in three patients. Two patients died due to brain stem invasion and secondary complications (8 and 11 months postoperatively). One patient died because of a concomitant hemophagocytic syndrome and hepatoblastoma (16 months postoperatively). When GOS scores were dichotomized into favorable (GOS $>3$ ) and unfavorable (GOS $\leq 3), 16(70 \%)$ had favorable outcome and seven $(30 \%)$ had unfavorable outcome. Six patients had reoperation, and five of those had favorable outcome after a mean follow-up of 8.6 years. Based on the univariate analysis, low histological grade $(p=0.030)$ and gross total resection $(p=0.024)$ were associated with favorable outcome. In addition, superficial location of tumor $(p=0.08)$ was a marginally significant variable for favorable outcome (Table III). One- and 10 -year overall survival rates were $91 \%$ and $86 \%$, respectively.

\section{Case Illustration 1}

A 7-year-old girl was brought to the Emergency Department with complaints of headache and somnolence. Cranial MRI revealed a T2-hyperintense, $49 \times 52 \times 53 \mathrm{~mm}$ mass lesion located in the pineal region that was consistent with a meningioma (Figure 2A-C). Tumor displaced both lateral ventricles laterally. A right-sided posterior interhemispheric

Table II: Chararcteristics of Patients with Tumor Relapses

\begin{tabular}{ccccc}
\hline $\begin{array}{c}\text { EOR at first } \\
\text { operation }\end{array}$ & Location & $\begin{array}{c}\text { Time to recurrence/ } \\
\text { progression (year) }\end{array}$ & Histology/Grade & $\begin{array}{c}\text { Total follow-up } \\
\text { (years) }\end{array}$ \\
\hline Gross total & Convexity & 5 & Transitional/I & 25.3 \\
\hline Gross total & Convexity & 4 & Transitional/I & 18.3 \\
\hline Gross total & Mesencephalo-thalamic & 1 & Atypical/II & 17.4 \\
\hline Gross total & Parasagittal & 5 & Atypical/II & 4.3 \\
\hline Subtotal & Retro-orbital & 4 & Transitional/I & 21.7 \\
\hline Subtotal & Spinal & 3 & Papillary/lII & 2.8 \\
\hline Subtotal & Retro-orbital & 3 & Transitional/I & 3.3 \\
\hline Subtotal & Anterior cranial fossa & 9 & Meningothelial/I & 15.3 \\
\hline Subtotal & Foramen magnum & 1 & Atypical/II & 0.7 \\
\hline Subtotal & Foramen magnum & 1 & Clear cell/II & 0.9 \\
\hline
\end{tabular}

EOR: Extent of resection. 
transfalcine approach is utilized. Due to increased intracranial pressure (ICP), an external ventricular catheter was placed through the Frazier's point to facilitate brain retraction. Histological examination revealed a WHO grade II atypical meningioma. Postoperative MRI shows gross total resection of the tumor. Despite the restricted diffusion in the left thalamus based on the postoperative diffusion-weighted imaging (DWI), the outcome of patient was excellent at 6-month follow-up assessment.

\section{Case Illustration 2}

A 16-year-old boy was admitted to our clinic with complaints of

Table III: Distribution of Dichotomized Outcomes and Their Relation to Patient and Tumor Characteristics. Data Represent Percentage [95\% Confidence Interval]

\begin{tabular}{lccc}
\hline Variable & Favorable & Unfavorable & $\mathbf{p}$ \\
\hline Sex (\%) & & & 0.538 \\
\hline Male & $83.3[33.7-75.4]$ & $72.7[24.6-66.3]$ & \\
\hline Female & $16.7[11.8-76.9]$ & $27.3[23.1-88.2]$ & \\
\hline Mean age at presentation (years) & $12.4[10.1-14.7]$ & $15.5[12.1-18.9]$ & 0.172 \\
\hline Neurofibromatosis (\%) & & & 0.292 \\
\hline Present & $40.0[21.9-61.3]$ & $16.7[3.0-56.4]$ & \\
\hline Absent & $60.0[38.7-78.1]$ & $83.3[43.6-97.0]$ & \\
\hline Tumor volume (cm $)$ & $71.8[26.2-117.3]$ & $28.2[1.8-34.7]$ & 0.300 \\
\hline Tumor location (\%) & & & 0.080 \\
\hline Superficial & $92.3[36.5-75.5]$ & $64.3[24.6-66.3]$ & \\
\hline Other & $7.7[3.0-56.4]$ & $35.7[23.1-88.2]$ & \\
\hline Extent of resection (\%) & & $\mathbf{0 . 0 2 4}$ \\
\hline$\quad$ Gross total & $89.5[68.6-97.1]$ & $50.0[21.5-78.5]$ & \\
\hline$\quad$ Subtotal & $10.5[2.9-31.4]$ & $50.0[21.5-78.5]$ & \\
\hline Histologic grade (\%) & & & $\mathbf{0 . 0 3 0}$ \\
\hline$\quad$ Grade I & $66.7[24.6-66.3]$ & $16.7[24.6-66.3]$ & \\
\hline$\quad$ Grade II, III & $33.3[24.6-66.3]$ & $83.3[24.6-66.3]$ & \\
\hline
\end{tabular}
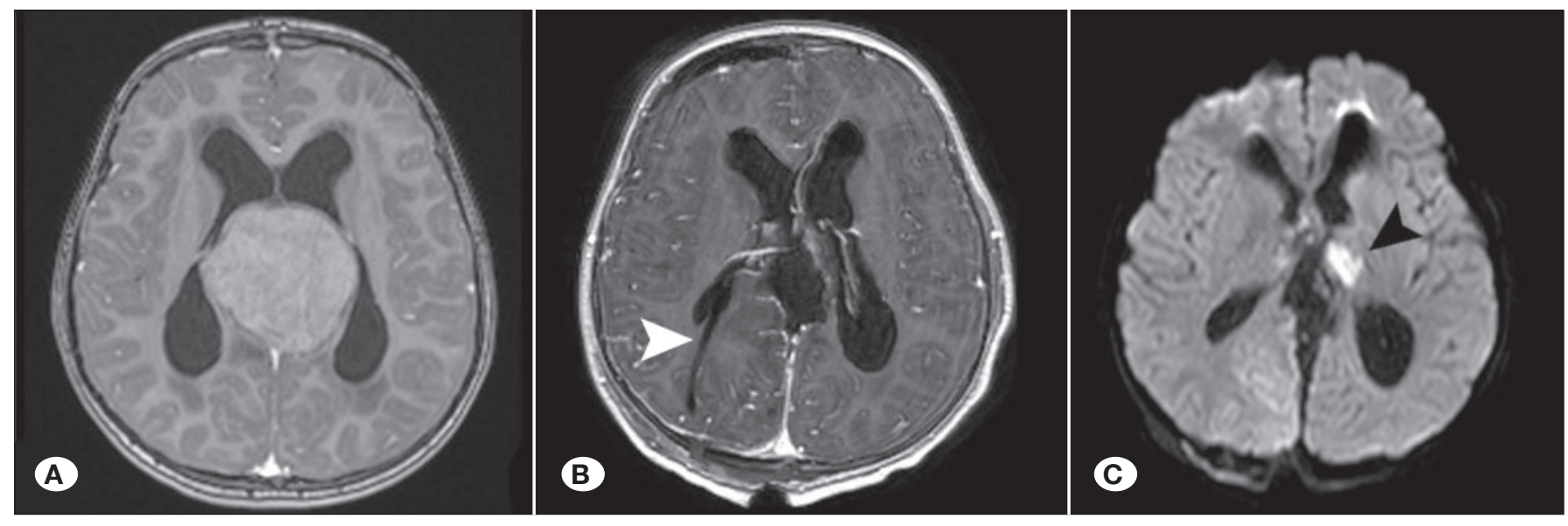

Figure 2: Preoperative contrast-enhanced axial (A), postoperative contrast-enhanced axial (B), and postoperative diffusion-weighted (C) images (DWI) of a 7-year-old girl. In order to facilitate posterior interhemispheric approach an external ventricular drainage catheter was introduced prior to retractor placement (white arrow head). Patient's outcome was excellent although DWI shows restricted diffusion in the left thalamus (black arrow head). 
headache and disfiguring calvarial thickening. On inspection, he had extensive hyperostosis from the forehead to the lambdoid suture. Cranial MRI revealed a giant $(100 \times 67 \times 50 \mathrm{~mm})$ bifrontal parasagittal meningioma (Figure $3 \mathrm{~A}-\mathrm{G}$ ), which compressed the cerebral sulci and displaced the corpus callosum inferiorly. The mass was associated with an extensive calvarial hyperostosis. Presence of bilateral vestibular schwannomas was consistent with NF2. After bicoronal skin incision, high-speed drill was utilized to mobilize the entire calvarial vault that incorporated the hyperostotic region. Bifrontal mass was excised gross totally together with the convexity dura, whereas the involved superior sagittal sinus segment was preserved. Histological examination revealed a meningothelial meningioma WHO grade I, with low mitotic index. After complete tumor excision and duraplasty with artificial dural substitute, the cranial vault was reconstructed using methyl methacrylate. The patient's neurological function was excellent with no recurrence or cosmetic deformity at 5-year follow-up.

\section{Case Illustration 3}

A 16-year old male admitted with nausea, vomitting and night sweats. The patient had history of hemophagocytic syndrome, CD19 deficiency and X-linked lymphoproliferative disase, which were diagnosed when he was 8-year old. On his last admission a hepatic ultrasound demonstrated three mass lesions, the biggest measuring $100 \times 57 \mathrm{~mm}$, which contained patchy necrotic areas. Excisional biopsy revealed hepatoblastoma and the patient received 4 cycles of chemotherapy. Due to progressive hepatic disease, liver transplantation was planned and during his routine preoperative work up, a right parietal, lobulated, extra-axial mass lesion which measured $24 \times 15 \mathrm{~mm}$ in maximal diameter (Figure 4A-D) suggestive of a metastatic lesion was demonstrated in cranial MRI. The tumor was excised totally, and the perioperative period was unremarkable. Histological examination of the tumor revealed meningothelial type meningioma WHO Grade I. Unfortunately, the patient died in $16^{\text {th }}$ month of follow due to febrile neutropenia.

\section{DISCUSSION}

Only $1.5 \%-1.8 \%$ of all meningiomas are pediatric meningiomas $(20,24)$. According to the Central Brain Tumor Registry of the United States, $2.5 \%$ of all primary pediatric CNS tumors originate from the meninges; however, this rate is $22 \%$ when all age groups are considered (20). A recent systematic review of pediatric meningioma similarly revealed that they represent $2.2 \%$ of all pediatric CNS tumors and $2.5 \%$ of all meningiomas (15). As our cohort constituted $2.5 \%$ of all pediatric CNS tumors and $1.7 \%$ of meningiomas in all age groups, the presented data is also in line with the reported literature confirming the rarity of these tumors during childhood.

Although meningiomas can be seen in all age groups, including the fetal period, its frequency increases after 20 years of age. When the population under 18 years is considered, the mean age at presentation for pediatric meningiomas is reported to be 8.1-13.7 years $(6,22,24,25)$. In our cohort, the mean presenting age (13.1 years) was similar to that of the general literature.
While adult meningiomas are seen predominantly in females, pediatric meningiomas have an opposite sex predilection (i.e., slightly more common in males). Differential expression of sex hormones or other pathogenetic processes may underlie this difference. However, no difference was observed in our series.

Family history, neurofibromatosis, and previous irradiation are considered as main risk factors for the development of meningioma. In our series, no patients had a history of cranial or spinal irradiation. However, nearly one-third of the patients had neurofibromatosis (one NF1, five NF2). In a meta-analysis including 677 patients from 35 series of pediatric and adolescent meningiomas, the prevalence of neurofibromatosis was $14.5 \%$, of which NF2 accounted for $10.2 \%$ and NF1 for $3.4 \%$; the type of neurofibromatosis of the remaining $0.9 \%$ was not documented. Meningioma associated with meningioangiomatosis occurred in three patients (15). Actually, we encountered one case of meningioangiomatosis in our database that was not associated with a meningioma.

The most common clinical feature for pediatric meningiomas is the presence of headache. Headache has been reported in up to $62 \%$ of patients $(14,18)$. With decreasing order of frequency, other presenting signs and symptoms include papilledema (55\%) and nausea and vomiting (53\%), which are, along with headache, indicative of increased ICP. Less commonly encountered signs and symptoms are cranial nerve deficits, visual disturbances, seizures, motor weakness, and scalp masses $(18,22)$. Similar to the literature, our patients mostly had signs of increased ICP, followed by seizures. Increased ICP is possibly due to fast-growing tumors in this age group.

Pediatric meningiomas tend to occur in atypical locations as compared with its adult counterparts. Although cerebral convexity (29.8\%) was the most common location, intraventricular meningiomas are far more common in children $(11 \%)$ than in adults $(1 \%-2 \%)$. Other common supratentorial locations included parafalcine or parasagittal (8.6\%), anterior and middle cranial fossa (7.2\%), and orbital and optic nerve sheath (4.9\%). Infratentorial (11.3\%) and spinal (5.6\%) meningiomas are relatively less common (15). In line with the literature, meningiomas in our cohort are most commonly located in the cerebral convexity $(25.0 \%)$ followed by the parasagittal region (21.4\%), whereas intraventricular meningioma (3.6\%) was less frequent compared with the results of other series $(15,18)$. Nevertheless, we have encountered meningiomas in other rare locations such as the pineal region or sylvian fissure.

Pediatric meningiomas usually present with larger sizes in comparison with its adult counterparts possibly due to rapid growth and/or delayed manifestation owing to developmental features (tolerability of cranial vault or inability to express symptoms). The mean tumor diameter was found to be 5.48 $\mathrm{cm}$ in 156 cases reviewed in a meta-analysis (15), and more than $45 \%$ of the pediatric meningiomas was reported to be bigger than $5 \mathrm{~cm}$ in another literature review (18). Similarly, out of 23 intracranial tumors in our series, $15(65 \%)$ were larger than $4 \mathrm{~cm}$ in diameter, and the mean maximal tumor diameter was $5.2 \pm 1.9 \mathrm{~cm}$ (median, $5 \mathrm{~cm}$; range $2.5-9.5 \mathrm{~cm}$ ). Although 

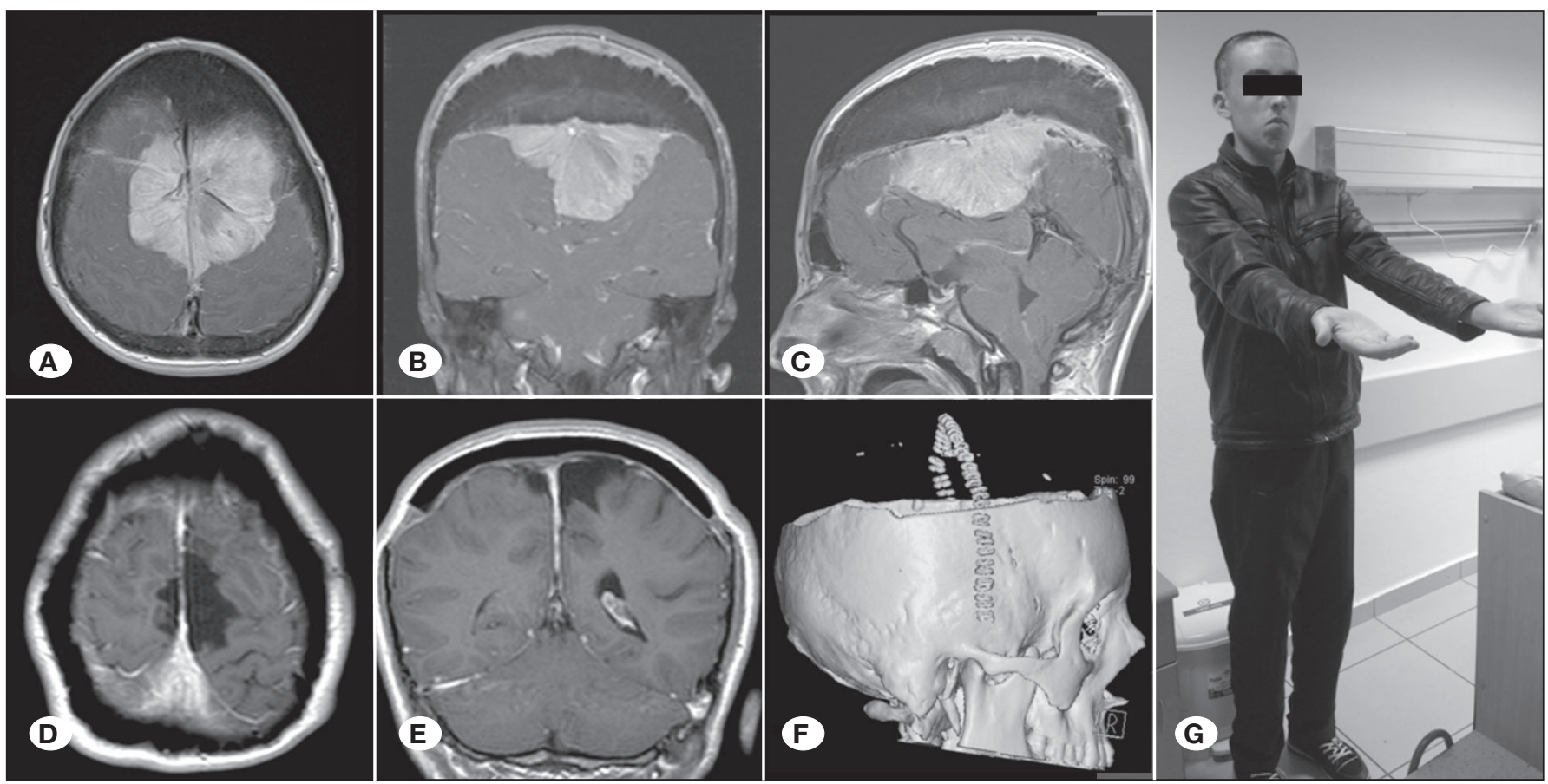

G.

Figure 3: Patient with bifrontal meningioma with accompanying extensive hypersosteosis. T1-weighted axial (A), coronal (B), and
sagittal (C) images show strong enhancement of 100x67x50 mm tumor as well as effacement of cerebral sulci and cerebellar folia. Postoperative images (D, E) show gross total resection of tumor that turned out to be meningothelial meningioma. Cranial vault had been reconstructed using methyl methacrylate, which is translucent in computed tomography images (F). Patient was without neurological deficit and displayed a good cosmetic result (G).
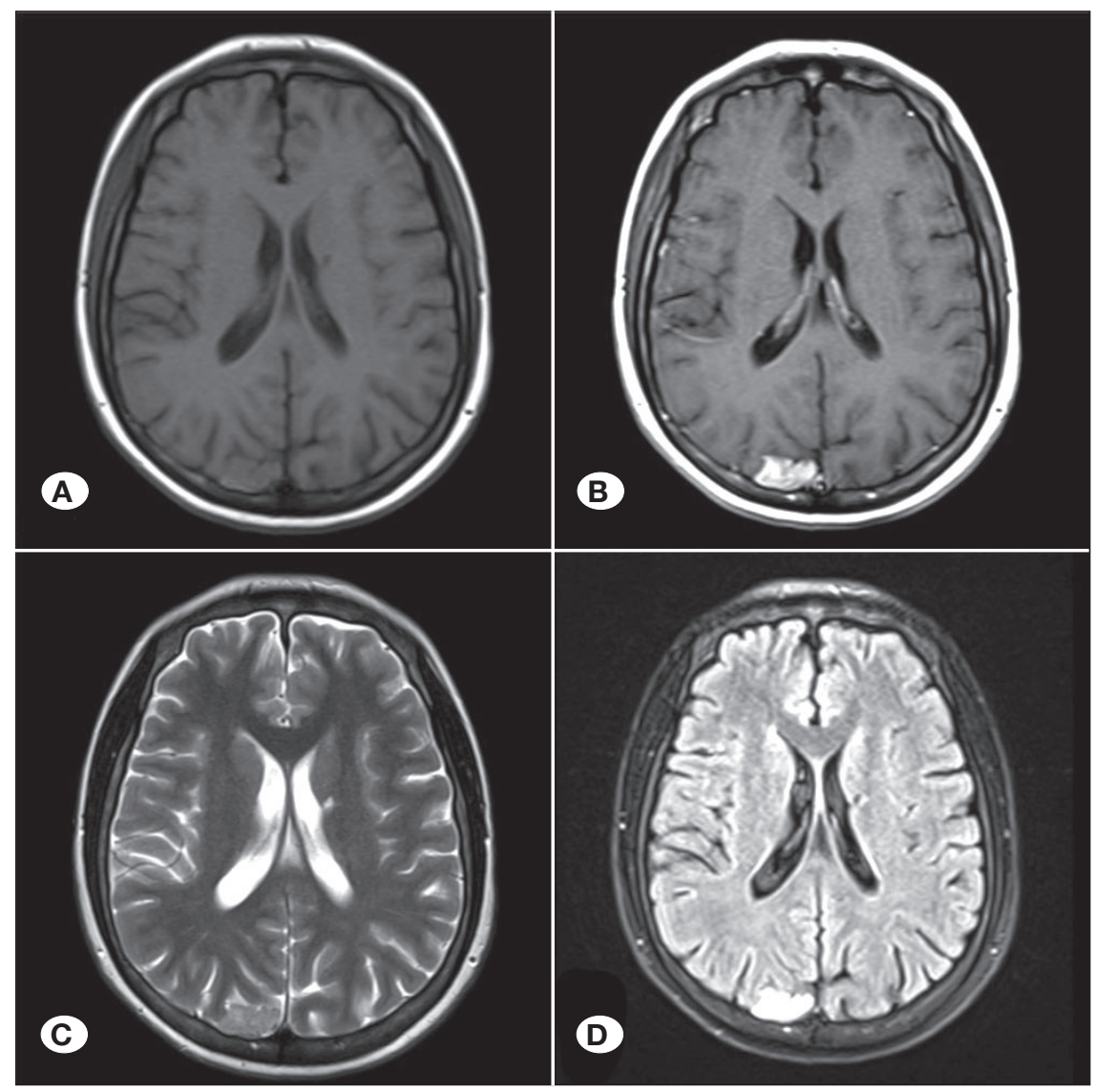

Figure 4: Axial T1-weighted (A), contrast enhanced T1-weighted (B), T2-weighted (C) and FLAIR (D) cranial magnetic resonance images of the patient described in Case Illustration 3. The lesion in right posterior parietal lobe is iso-intensewith cortex in T2 and hypo-intense in T1-weighted images.

There is strong contrast enhancement without dural tail sign; however, brain parenchymathat surrounds the lesion does not show any sign of edeuma in T2 and FLAIR images. Radiological differential diagnosis included metastasis of the primary lesion and solitary fibrous tumor of dural origin. The imaging characteristics are very atypical for classical meningioma. 
other MRI characteristics of adult and pediatric meningiomas were generally similar, dural tail sign is less and intratumoral cysts are more common in pediatric meningiomas $(2,7,12)$.

It is a well-known fact that higher-grade (II or III) meningiomas occur more frequently in children and young male adults than in older adults (26). A meta-analysis of published pediatric meningioma series showed that although $\mathrm{WHO}$ grade I meningiomas comprised $79 \%$ of them, WHO grade II (10\%) and grade III (9\%) were also relatively common. In our series, the distribution of grades I (55.5\%), II (33.3\%), and III (11.2\%) meningiomas confirmed much higher rates of advanced grade in pediatric meningiomas as in some other recently published series $(7,10,11)$.

Surgical resection is the mainstay of meningioma management with complete tumor excision being the best first-line treatment option to achieve favorable short- and long-term prognosis. Although common principles of meningioma surgery apply to childhood meningiomas in general, several features make them more challenging compared with adult meningiomas. First, they tend to occur in atypical locations, usually deep-seated and adjacent to critical structures. Second, they tend to present with larger tumors. Third, histologically aggressive forms are more common in children. Fourth, diffuse and anatomically aggressive or invasive forms are also more common as noted in our series. An important and wellknown feature of meningiomas is associated reactive hyperostosis. Notably, the hyperostosis and its consequences are seldom reported in recent literature $(1,12)$. In our experience, those tumors are technically challenging due to bone anatomy, sinus invasion, tumor vascularity, and pial adherence or brain invasion. Fifth, pediatric meningiomas require aggressive surgical treatment taking into account longer life expectancy, higher recurrence risk, and detrimental effects of other treatment modalities (radiotherapy, chemotherapy). In addition, although meningioma is better than other childhood tumors, surgery is not without morbidity. Hence, careful preoperative planning with different imaging modalities (MRI, computed tomography, angiography); technical preparation and intraoperative adjunct use (preoperative embolization if feasible, neuronavigation, intraoperative neuromonitoring); meticulous surgical technique including appropriate bone drilling, use of cavitron ultrasonic surgical aspirator, and careful microsurgical dissection yield complete resection with low morbidity and excellent long-term outcomes in those technically challenging tumors.

Adjuvant treatment is not considered a good option in most pediatric meningiomas. Although some pediatric oncology guidelines recommend radiotherapy for grade III meningiomas, upfront radiotherapy has not been found to provide significant survival benefit in pediatric meningioma cases among similar subgroups of tumor grade or initial resection status (15). Although more aggressive when compared with adult forms, survival with these tumors are long with radical surgery; radiotherapy is usually delayed until repeated surgeries fail to be beneficial in order to avoid longterm consequences since these patients are in childhood age. In our experience, only five patients with subtotally removed, higher-grade, and recurrent tumors received radiotherapy. We believe that early aggressive surgical excision must be the primary treatment option for pediatric meningiomas of all grades; and even residual progression or recurrences should be treated with surgery (five out of six patients with repeat surgery had favorable long-term outcomes in the present series). Radiotherapy should be reserved only for those patients with rapidly progressing or recurring higher-grade (II or III) meningiomas (4). Moreover, chemotherapy is not an effective treatment option for meningiomas, and experience with chemotherapy in pediatric meningiomas is extremely limited in the literature.

An average follow-up period between 2.8 and 8.5 years for pediatric meningiomas has been reported in the literature $(1,6,8,9,16,17,19,20,22,25)$. In this regard, the present study is among the few studies with a long-term follow-up (mean, $10.3 \pm 7.7$ years; median, 9 years). We observed three patients expired and two lost to follow-up in that period. In general, meningioma progression is relatively slow. Therefore, it is imperative to have longer follow-up to establish functional status data of these children in adult life and identify relapses properly. In addition long-term sequelae of pediatric meningioma survivors should not be overlooked, which include visual deficits, neuropsychological abnormalities, developmental delay, CNS palsies, motor deficits, seizure disorders, and sensory deficits (14).

Low- or high-grade pediatric meningiomas are not exempt from relapses. Indeed, five (33.3\%) of $15 \mathrm{WHO}$ grade I and five $(41.7 \%)$ of $12 \mathrm{WHO}$ grade II or III meningioma patients in our study experienced relapses. Latencies for relapses in low- and high-grade groups were $5.0 \pm 2.9$ years and $2.2 \pm 2.1$ years, respectively. Similarly, Grossbach et al. reported that in their 39-patients series with WHO grade I tumors, the recurrence rate was $42 \%$ (10 of 24 ); this rate was not significantly different from the recurrence rate among patients with WHO grade II or III pathology (10). Presented data in line with their findings suggest that even WHO grade I meningiomas in children may behave more like adult atypical meningiomas than classic adult meningothelial meningiomas, indicating different biological behavior pattern.

In the present series, we identified WHO grade I, convexity or parasagittal location, and gross total resection as favorable prognostic factors. Risk of tumor recurrence and, thus, poor outcomes increase with histological grade both in adults and children. Interestingly, children and adolescents with meningioma have better progression-free and overall survival rates than adults despite the fact that they harbor more aggressive tumors based on WHO grading (15). This fact implies that $\mathrm{WHO}$ grade alone is not sufficient in determining biological behavior of pediatric meningiomas and that they may have different molecular characteristics from adult meningiomas. The extent of resection has also been a wellestablished independent prognostic factor of meningiomas (23). However, with regard to the location, literature is controversial. On one hand, Celtikci et al. suggested that convexity or nonskull base meningiomas are more frequently associated with higher grades, tumor recurrence, progression, 
and worse prognosis in adults (5). On the other hand, skull base or deep meningiomas are associated with higher surgical morbidity and lower complete excision rates even though they may have less aggressive behavior, thus yielding similar recurrence and long-term outcomes (21). Likewise, a metaanalysis of pediatric meningiomas did not find a relationship between tumor location and progression-free or overall survival (15).

Herein, we have presented a study describing various characteristics in 23 patients harboring one or more meningioma. We sought to define variables that are associated with favorable outcome. Although we have shown that both extent of resection and histological grade are related to better outcomes and a marginal advantage of convexity and parasagittal tumors in univariate analysis is noted, the relatively small number of cases in our study precluded a multivariate analysis, which we believe is one of the most important weaknesses of our study. Nevertheless, our series is one of the largest cohorts with long-term follow-up data in the MRI era. Another drawback of our study is its retrospective design; however, the reader would admit the difficulty in collecting prospective data, which is due to the rarity of the disorder within a relatively long duration. It is possible that the higher recurrence rates we observed are in fact related to the extent of initial resection and actually represent tumor progression. This is especially relevant with regard to the earlier cases from the pre-MRI era in which verification of the extent of resection was extremely limited. Thus, it is difficult to distinguish true recurrence from progression in this series (10).

\section{CONCLUSION}

Meningiomas in pediatric age group have important differences from its adult counterparts such as higher incidence of atypical locations, grade II or III, and larger tumors that pose challenges in terms of management. Nevertheless, prognosis of meningiomas, even of higher grades, is relatively better when compared with other high-grade pediatric tumors. Early aggressive surgical resection is the best treatment option and yields favorable early and long-term outcomes. Recurrences should also be treated with surgery if possible, and radiotherapy should only be reserved for rapidly progressive or recurrent high-grade meningiomas for which surgical treatment fails to achieve optimal disease control.

\section{- REFERENCES}

1. Alexiou GA, Mpairamidis E, Psarros A, Sfakianos G, Prodromou N: Intracranial meningiomas in children: Report of 8 cases. Pediatr Neurosurg 44:373-375, 2008

2. Arivazhagan A, Devi BI, Kolluri SV, Abraham RG, Sampath S, Chandramouli BA: Pediatric intracranial meningiomas-do they differ from their counterparts in adults? Pediatr Neurosurg 44:43-48, 2008

3. Bilginer B, Hanalioglu S, Turk CC, Narin F, Oguz KK, Soylemezoglu F, Akalan N: Is the knowledge pertaining to adult glioblastomas enough for pediatric cases? prognostic factors in childhood. Turk Neurosurg 27:279-288, 2017
4. Bilginer B, Turk CC, Narin F, Hazer B, Hanalioglu S, Oguz KK, Soylemezoglu F, Akalan N: De novo formation of brain tumors in pediatric population following therapeutic cranial irradiation. Childs Nerv Syst 31:893-899, 2015

5. Celtikci E, Kaymaz AM, Akgul G, Karaaslan B, Emmez $\mathrm{OH}$, Borcek A: Retrospective analysis of 449 intracranial meningioma patients operated between 2007 and 2013 at a single institute. Turk Neurosurg 28:1-6, 2018

6. El Beltagy MA, Enayet AE, Atteya MME, Reda M, Refaat A, Taha H, Ahmed S, Abdelaziz A: Management of pediatric CNS meningiomas: CCHE-57357 experience in 39 cases. Childs Nerv Syst 35:1323-1331, 2019

7. Fan MC, Fang W, Liu K, Wang C, Deng WS, Sun P, Tang WZ: Paediatric intracranial meningiomas: Eight-year experience with 32 cases. Chinese Neurosurgical Journal, 2017

8. Gao X, Zhang R, Mao Y, Wang Y: Childhood and juvenile meningiomas. Childs Nerv Syst 25:1571-1580, 2009

9. Greene S, Nair N, Ojemann JG, Ellenbogen RG, Avellino AM: Meningiomas in children. Pediatr Neurosurg 44:9-13, 2008

10. Grossbach AJ, Mahaney KB, Menezes AH: Pediatric meningiomas: 65-year experience at a single institution. $\mathrm{J}$ Neurosurg Pediatr 20:42-50, 2017

11. Huntoon K, Pluto CP, Ruess L, Boue DR, Pierson CR, Rusin JA, Leonard J: Sporadic pediatric meningiomas: A neuroradiological and neuropathological study of 15 cases. $J$ Neurosurg Pediatr 20:141-148, 2017

12. Im SH, Wang KC, Kim SK, Oh CW, Kim DG, Hong SK, Kim NR, Chi JG, Cho BK: Childhood meningioma: Unusual location, atypical radiological findings, and favorable treatment outcome. Childs Nerv Syst 17:656-662, 2001

13. Isikay I, Hanalioglu S, Basar I, Narin F, Bilginer B: Survival benefit with gross total resection and adjuvant radiotherapy in childhood atypical teratoid/rhabdoid tumors: Results of a single-center cohort of 27 cases. Turk Neurosurg 29:689-697, 2019

14. Kotecha RS, Junckerstorff RC, Lee S, Cole $\mathrm{CH}$, Gottardo NG: Pediatric meningioma: Current approaches and future direction. J Neurooncol 104:1-10, 2011

15. Kotecha RS, Pascoe EM, Rushing EJ, Rorke-Adams LB, Zwerdling T, Gao X, Li X, Greene S, Amirjamshidi A, Kim SK, Lima MA, Hung PC, Lakhdar F, Mehta N, Liu Y, Devi BI, Sudhir BJ, Lund-Johansen M, Gjerris F, Cole CH, Gottardo NG: Meningiomas in children and adolescents: A meta-analysis of individual patient data. Lancet Oncol 12:1229-1239, 2011

16. Lakhdar F, Arkha Y, El Ouahabi A, Melhaoui A, Rifi L, Derraz S, El Khamlichi A: Intracranial meningioma in children: different from adult forms? A series of 21 cases. Neurochirurgie 56:309314, 2010

17. Li X, Zhao J: Intracranial meningiomas of childhood and adolescence: Report of 34 cases with follow-up. Childs Nerv Syst 25:1411-1417, 2009

18. Liu Y, Li F, Zhu S, Liu M, Wu C: Clinical features and treatment of meningiomas in children: Report of 12 cases and literature review. Pediatr Neurosurg 44:112-117, 2008

19. Mehta N, Bhagwati S, Parulekar G: Meningiomas in children: A study of 18 cases. J Pediatr Neurosci 4:61-65, 2009 
20. Menon G, Nair S, Sudhir J, Rao BR, Mathew A, Bahuleyan B: Childhood and adolescent meningiomas: A report of 38 cases and review of literature. Acta Neurochir (Wien) 151:239-244; discussion 244, 2009

21. Nanda A, Vannemreddy P: Recurrence and outcome in skull base meningiomas: Do they differ from other intracranial meningiomas? Skull Base 18:243-252, 2008

22. Santos MV, Furlanetti L, Valera ET, Brassesco MS, Tone LG, de Oliveira RS: Pediatric meningiomas: A single-center experience with 15 consecutive cases and review of the literature. Childs Nerv Syst 28:1887-1896, 2012
23. Simpson D: The recurrence of intracranial meningiomas after surgical treatment. J Neurol Neurosurg Psychiatry 20:22-39, 1957

24. Stanuszek A, Piatek P, Kwiatkowski S, Adamek D: Multiple faces of children and juvenile meningiomas: A report of single-center experience and review of literature. Clin Neurol Neurosurg 118:69-75, 2014

25. Thuijs NB, Uitdehaag BM, Van Ouwerkerk WJ, van der Valk $P$, Vandertop WP, Peerdeman SM: Pediatric meningiomas in The Netherlands 1974-2010: A descriptive epidemiological case study. Childs Nerv Syst 28:1009-1015, 2012

26. Zhou P, Ma W, Yin S, Li Y, Jiang S: Three risk factors for WHO grade II and III meningiomas: A study of 1737 cases from a single center. Neurol India 61:40-44, 2013 